\title{
Integrin-Cytoskeletal Interactions in Neuronal Growth Cones
}

\author{
Christine E. Schmidt, ${ }^{1}$ Jianwu Dai, ${ }^{3}$ Douglas A. Lauffenburger, ${ }^{1,2}$ Michael P. Sheetz, ${ }^{3}$ and Alan F. Horwitz ${ }^{2}$ \\ Departments of ${ }^{1}$ Chemical Engineering and ${ }^{2}$ Cell and Structural Biology, University of Illinois, Urbana, Illinois 61801 \\ and ${ }^{3}$ Department of Cell Biology, Duke University Medical Center, Durham, North Carolina 27710
}

\begin{abstract}
Development of the nervous system requires that neuronal growth cones, in coordination with growing axons, migrate along precise paths defined by specific extracellular matrix cues until they encounter their targets. Laminin promotes growth cone migration through receptors such as the integrins, but the underlying physical mechanism is poorly understood. We have Investigated the cytoskeletal associations and surface dynamics of endogenous $\beta 1$ integrins in chick dorsal root ganglion growth cones migrating on laminin. A single-beam optical gradient trap was used to place 0.5 - $\mu \mathrm{m}$-diameter polystyrene beads conjugated with anti- $\beta 1$ integrin monoclonal antibodies at desired locations on the growth cone surface. We found a substantial increase in the stable attachment of these beads, with subsequent slow rearward motion, on the front periphery of the growth cone compared to the base. The surface dynamics of smaller aggregates of integrin were explored by monitoring the temporal and spatial displacements of $40-\mathrm{nm}$ diameter gold particles coated with anti-ß1 integrin antibodies. The small particles were transported preferentially to the growth cone periphery by brief directed excursions interspersed with periods of diffusion. In addition, the leading edge of the growth cone was supported to a greater extent by an actin-dependent cytoskeleton that resisted mechanical tether formation. Such a regional differentiation of the growth cone has not been documented previously and has implications for the mechanism of growth cone migration and guidance.
\end{abstract}

[Key words: nerve growth cone, migration, motility, integrin, cytoskeleton, laser optical trap, single-particle tracking]

The axonal growth cone migrates in response to various recognition signals over long distances, guiding the extending axon along precise pathways to its final destination (Trinkaus, 1984; Bray and Hollenbeck, 1988). Growth cone guidance involves both stimulatory and inhibitory cues, which are perceived by filopodia and lamellipodia extending in all directions from the distal tip of the axon. Guidance information consists of soluble, diffusible factors that act as chemoattractants (Tessier-Levigne

\footnotetext{
Received Oct. 4, 1994; accepted Nov. 8, 1994.

We are extremely grateful to Chris Martenson for his generous help with the dissections and to Ken Yamada for providing the hybridoma that produces the ES66 monoclonal antibody. This work was supported by National Institutes of Health grants GM07283 (Cell and Molecular Biology Training Grant) to C.E.S., GM23244 to A.F.H., GM36277 and a grant from Human Frontiers in Science Program to M.P.S.

Correspondence should be addressed to Professor Douglas A. Lauffenburger Center for Biomedical Engineering, MIT, 66-446, Cambridge, MA 02139.

Copyright $(\mathcal{C} 1995$ Society for Neuroscience $0270-6474 / 95 / 153400-08 \$ 05.00 / 0$
}

and Placzek, 1991) or chemorepellents (Pini, 1993), as well as insoluble cell-cell or cell-substrate signals (Rutishauser, 1993). With regard to the latter, cell adhesion molecules (CAMs), including the neural cell adhesion molecule (NCAM) (Cunningham et al., 1987; Walsh and Doherty, 1991), N-cadherin (Takeichi, 1988), and LI (Seilheimer and Schachner, 1988), mediate cell-cell interactions, while integrins promote the adhesion of neurons to extracellular matrix (ECM) molecules such as laminin (Bozyczko and Horwitz, 1986; Reichardt and Tomaselli, 1991). Together, cell-cell and cell-matrix interactions support the adhesion and migration of growth cones over different matrices and cell types, including Schwann cells and muscle cells.

The actin cytoskeleton, which is prominent in filopodia and lamellipodia of the axon's distal tip, is critical for generating the forces necessary for growth cone crawling (Bentley and O'Connor, 1994). The rearward flow of actin filaments, likely the result of contributions from both spatially localized actin polymerization and depolymerization processes (Forscher and Smith, 1988; Okabe and Hirokawa, 1991) and actin-myosin interactions (Koch, 1980; Letourneau, 1981), provides possible mechanisms for the force developed by the growth cone against its substratum. Transmission of internal movements of actin filaments into a force pulling the growth cone forward also requires an adhesive interaction of the cell with its surroundings (Heidemann et al., 1990), as mediated by transmembrane adhesion receptors such as the integrins that link the internal cytoskeletal network to ECM proteins (Bozyczko and Horwitz, 1986; Reichardt and Tomaselli, 1991).

The actin cytoskeleton and adhesive contacts are also vital for appropriate path selection of the neuron (Bentley and O'Connor, 1994). In fact, it is the turning of the growth cone that will deterninie the future trajectory of the extending axon, and hence the ultimate synaptic target (Bray, 1987; Mitchison and Kirschner, 1988; Gordon-Weeks, 1989). Growth cone steering requires the formation of stable adhesive contacts, as mediated by adhesion receptors, with matrix proteins along the selected path or with epithelium or high-affinity guidepost cells. Such contacts result in the local accumulation of F-actin (Lin and Forscher, 1993; O'Connor and Bentley, 1993), which subsequently "caps" unstable microtubules extending from the axon into the proximal portion of the growth cone (Gordon-Weeks, 1991; Sabry et al., 1991; Tanaka and Kirschner, 1991). This stabilization then results in reorganization (consolidation) of microtubules and reorientation of the axon. Adhesive contacts also appear to modulate directional changes of the motile growth cone via the tension generated by attachment of actin-rich filopodia (Bray, 1979). In addition, several lines of evidence show that tension applied directly to the axon can both stimulate neurite growth and direct its elongation (Bray, 1984; Zheng et al., 1991). 
The focus of the present work is on the adhesive interactions that contribute to the processes leading to growth cone guidance. In particular, we are interested in how the associations of $\beta 1$ integrin with internal cytoskeletal elements can help regulate growth cone migration and steering. We have used laser optical trapping and nanometer-precision tracking analyses to monitor the coupling of $\beta 1$ integrin, a family of integrins that mediates attachment of the growth cone to laminin, to the actin cytoskeleton. In addition, we have utilized the laser tweezers to monitor the mechanical properties of membrane tethers that are sensitive to the linkage between the cytoskeleton and the membrane. Our findings suggest that at least some aspect of growth cone locomotion and steering, and hence axonal pathfinding, may be regulated at the level of integrin-cytoskeleton coupling.

\section{Materials and Methods}

Cell culture. Dorsal root ganglion (DRG) explants were dissected from 12 d chick embryos (Okun, 1972) and plated in growth wells on treated coverslips (see below). Explants were maintained at $37^{\circ} \mathrm{C}, 5 \% \mathrm{CO}_{2}$ in culture medium: clear MEM (GIBCO-Bethesda Research Labs, Grand Island, NY) supplemented with glucose to $6 \mathrm{gm} / \mathrm{liter}, 20 \mathrm{mM}$ HEPES, $2 \mathrm{mM}$ L-glutamine, $50 \mathrm{U} / \mathrm{ml}$ penicillin and streptomycin, $\mathrm{N} 2$ serum-free supplement (GIBCO), and $10 \mathrm{ng} / \mathrm{ml}$ nerve growth factor $(2.5 \mathrm{~S}$; GIB$\mathrm{CO}$ ). The explants were incubated for at least $24 \mathrm{hr}$ before use and medium was changed every $48 \mathrm{hr}$. Prior to observation, the cloning cylinder was removed and the coverslip containing the cells was mounted on an aluminum coverslip holder using silicone grease. Twenty microliters of the appropriate bead solution diluted in warmed culture medium were added to the cells, and then a second glass coverslip was mounted on top. The unit was sealed at the corners with melted valap (Vaseline, lanolin, and paraffin, 1:1:1).

Coverslip and substrate preparation. No. 1 glass coverslips, $22 \mathrm{~mm}^{2}$ (VWR, San Francisco, CA), and 10-mm-diameter glass cloning cylinders (Bellco, Vineland, $\mathrm{NJ}$ ) were cleaned by soaking in $20 \% \mathrm{HNO}_{3}$ for $30 \mathrm{~min}$, followed by rinsing in distilled water overnight. Coverslips and cylinders were sterilized by soaking in $70 \%$ ethanol and drying in a sterile laminar flow hood. To prepare the cell growth wells and to aid in centering the explant, a cloning cylinder was secured to the coverslip with sterilized silicone grease. The growth wells were coated with $0.01 \%$ poly-D-lysine for $30 \mathrm{~min}$ at room temperature, rinsed three times with sterile water, then allowed to dry in a sterile hood. The day of the dissection, poly-D-lysine-coated coverslips were exposed for $15 \mathrm{~min}$ to a 1:50 Matrigel (Collaborative Research, Bedford, MA): MEM solution.

Bead preparation. ES66-8 rat hybridoma cells were generously provided by Dr. Kenneth Yamada (NIH, Bethesda, MD). ES66 is a nonadhesion-perturbing monoclonal antibody directed against the chicken $\beta 1$ integrin (Duband et al., 1988). Antibody was purified from hybridoma supernatants by ammonium sulfate precipitation, followed by anion-exchange chromatography (Duband et al., 1988). Bovine serum albumin (BSA) and rat IgG (Sigma, St. Louis, MO) were used as control proteins in all experiments. ES66, rat IgG, and BSA were either adsorbed to $40-\mathrm{nm}$-diameter colloidal gold particles or chemically conjugated via a carbodiimide linkage to the surfaces of activated $0.5-\mu \mathrm{m}$ diameter polystyrene (latex) beads.

For gold bead preparations, ES66 $\mathrm{mAb}$ was diluted to a final concentration of $0.1 \mathrm{mg} / \mathrm{ml}$ in $0.5 \mathrm{ml}$ of calcium/magnesium-free phosphate-buffered saline (CMF-PBS). Then, $0.5 \mathrm{ml}$ of 40-nm (diameter) gold bead suspension (EY Laboratories Inc., San Mateo, CA) was added to the antibody solution and incubated on ice for $5 \mathrm{~min}$. For BSA-coated beads, $0.5 \mathrm{ml}$ of gold bead suspension was incubated on ice for $5 \mathrm{~min}$ with $0.5 \mathrm{ml}$ of $5 \mathrm{mg} / \mathrm{ml}(0.5 \%)$ BSA. After incubation with either ES66 or BSA, the beads were washed three times with $0.5 \%$ BSA in CMFPBS by centrifugation at $20,000 \mathrm{rpm}$ and $4^{\circ} \mathrm{C}$ for $10 \mathrm{~min}$ in a Beckman TL- 100 ultracentrifuge using a TLS-55 swinging bucket rotor (Beckman Instruments, Palo Alto, CA). The final bead pellet was resuspended in $50 \mu \mathrm{l}$ of culture medium and stored at $4^{\circ} \mathrm{C}$. The beads were diluted $1: 3$ in culture medium and sonicated just prior to their use.

For latex bead preparations, ES66 mAb was prepared at a concentration of $2 \mathrm{mg} / \mathrm{ml}$ in $50 \mu \mathrm{l}$ of CMF-PBS. Then, $25 \mu \mathrm{l}$ of $0.5 \mu \mathrm{m}$ Covaspheres $M X$ reagent (Duke Scientific, Palo Alto, CA) was added to the $\mathrm{mAb}$ solution and allowed to incubate for $75 \mathrm{~min}$ at room temperature. For BSA-coated beads, $25 \mu$ l of the latex bead reagent was in- cubated with $50 \mu \mathrm{l}$ of $0.5 \%$ BSA. After incubation with either ES66 or BSA, the beads were washed three times with $0.5 \%$ BSA in CMF-PBS by centrifugation at $10,000 \mathrm{rpm}$ and $4^{\circ} \mathrm{C}$ for $10 \mathrm{~min}$ in a microcentrifuge (Beckman Instruments). The final bead pellet was resuspended in $75 \mu \mathrm{l}$ of $0.5 \%$ BSA. Each bead solution was diluted 3:1000 in culture medium, then sonicated prior to being added to the cells.

To prepare IgG-coated latex beads, rat IgG was solubilized at a concentration of $10 \mathrm{mg} / \mathrm{ml}$ in PBS. Then, $50 \mu \mathrm{l}$ of Covaspheres MX reagent was added to $50 \mu \mathrm{l}$ of the above IgG solution and incubated overnight at $4^{\circ} \mathrm{C}$. The beads were washed three times with $\mathrm{MEM}$ by centrifugation at $10,000 \mathrm{rpm}$ and $4^{\circ} \mathrm{C}$ for $10 \mathrm{~min}$. The final bead pellet was resuspended in $100 \mu \mathrm{l}$ of MEM. For the experiments, the bead solution was diluted 3:100 in culture medium.

Video microscopy. Cells were viewed by high-magnification, videoenhanced differential interference contrast microscopy (IM-35, Zeiss, Oberkochen, Germany) with a fiber optic illuminator. The microscope stage was maintained at $37^{\circ} \mathrm{C}$ using an air current incubator. Images were collected with a video camera (VE1000, Dage-MTI Inc., Wabash, MI) and stored on half-inch s-VHS videotape using an s-VHS VCR (AG-7300, Panasonic, Secaucus, NJ). Images were digitized at video rate, $30 \mathrm{~Hz}$, from the videotape using a series 151 Image Processor (Imaging Technology, Inc., Woburn, MA), an IBM PC/AT-compatible computer (Zenith Z386/25 with $150 \mathrm{Mb}$ hard disk), and a real-time storage system (model 8300, Applied Memory Technology, Tustin, CA) A time-base corrector (FA-300, FOR-A, Boston, MA) was used to synchronize the video image timing signal prior to digitization by the image processor.

Analysis of gold particle position. Positions of the selected gold beads in the digitized images were determined for each video frame by the computer and image processor using the cross-correlation method of Gelles et al. (1988). The accuracy of tracking $40 \mathrm{~nm}$ gold particles using cross-correlation analysis was estimated as $\pm 5-10 \mathrm{~nm}$. Beads were tracked at video rate, $30 \mathrm{frames} / \mathrm{sec}$, over a total time of $100 \mathrm{sec}$.

Directed versus diffusive motion was determined statistically as previously described (Schmidt et al., 1993). Briefly, we calculated the probability that a diffusing particle will traverse at least a certain distance in a given direction $X$ simply by executing a random walk. The $X$ direction, that is, the general direction of motion for the bead, was determined by a least-squares fit of the two-dimensional position data for the bead. The displacement that each gold particle traversed in the $\mathrm{X}$ direction for the total tracking time of $100 \mathrm{sec}$ was determined from the bead trajectory and compared to the distance predicted for a purely diffusing particle. The statistical probability that a particle undergoing a random walk will diffuse from $x=0$ to a distance between $x$ and $x$ $+d x$ is described by the equation $P(x) d x=1 /(4 \pi D t)^{1 / 2} \exp \left(-x^{2} / 4 D t\right) d x$ (Berg, 1983), where $t$ is the time interval over which the net displacement, $x$, is measured and $D$ is the diffusion coefficient (see below). A cutoff for $P(x)$ of 0.05 was used: particle displacements having a probability of $5 \%$ or more as resulting from diffusion $[P(x) \geq 0.05]$ were scored as diffusive, and those with $P(x)<0.05$ were considered directed.

Values for the time-averaged velocity of directed motion, $\langle v\rangle$, and the diffusivity, $D$, were extracted from plots of parallel and two-dimensional mean-square displacement (MSD) versus timle plots as previously outlined (Sheetz et al., 1989; Schmidt et al., 1993). The average velocity for directed motion, $\langle v\rangle$, was obtained from a fit of the parallel MSD data to the function MSD $=2 D t+(v t)^{2}$ and the diffusion coefficient $D$, was determined from plots of two-dimensional MSD versus time, $\mathrm{MSD}=4 D t+(v t)^{2}$. Since these relationships assume a constant velocity imposed on the particle, the reported $\langle v\rangle$ is a time-averaged value over the entire particle trajectory.

An average value for the instantaneous velocity, $v_{i}$, of individual jumps was determined directly from the experimental integrin trajectories. The lengths of path segments that represented obvious rapid excursions, as determined using the same statistical methods mentioned above, and the time intervals for the excursions were used to calculate the instantaneous velocity, $v_{i}$. Only those directed intervals occurring over a period of $1-2$ sec were used to minimize potential noise from the experimental tracking routine.

Laser optical trapping. The laser trap consists of a polarized beam from a $1 \mathrm{~W}$ TEM $_{(x)}$-mode near-infrared $(1064 \mathrm{~nm}$ ) laser (model C-95, CVI Corporation, Albuquerque, NM) that is passed through a $3 \times$ beam expander (CVI Corporation), then focused through an $80 \mathrm{~mm}$ focal length achromat lens (Melles Griot, Irvine, CA) into the epifluorescence port of the Zeiss IM-35 microscope (Kuo et al., 1991). 


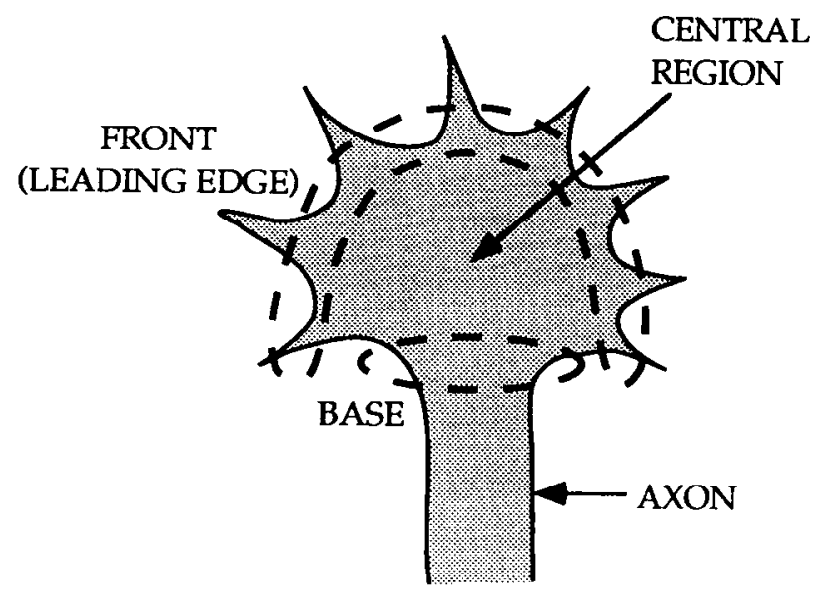

Figure 1. Locations on growth cone used for laser optical trapping experiments. The dashed enclosures indicate the front and base portions of the growth cone used for laser trapping. The growth cone periphery or leading edge (FRONT) was defined as active lamellar regions and portions of the membrane between extending filopodia (within $\sim 1-2$ $\mu \mathrm{m}$ of the edge). Trapping could not be performed directly on the filopodia because they are very thin making it difficult to avoid unwanted attachment of the bead to the slide surface. The base of the growth cone was identified as the proximal portion of the growth cone separating the actin-rich filopodia and lamellipodia from the microtubule-rich axon. The central region of the growth cone and the axon are as depicted in the drawing.

Polystyrene beads coated with BSA or anti-integrin mAb (ES66) were trapped with $\sim 50-60 \mathrm{~mW}$ of laser light from solution and held on either the front periphery or the base of the growth cone (see Growth cone selection, below, and Fig. 1) for $10 \mathrm{sec}$ to allow for interaction of the mAbs on the bead with integrin in the cell membrane. After a 10 $\mathrm{sec}$ hold of the bead on the cell surface, the trap was subsequently used to classify the behavior of the underlying integrin as either cytoskeletalattached or non-cytoskeletal-attached. An inability to move a bead with the trap indicated that the integrins bound by the bead were stably interacting with the underlying actin cytoskeletal network while those beads that could be pulled around within the plane of the membrane were scored as noncytoskeletal (Kucik et al., 1991; Schmidt et al., 1993).

To investigate general "tether" formation, IgG-coated beads were held with the laser trap $(\sim 60 \mathrm{~mW})$ on the cell surface for $5 \mathrm{sec}$ and then pulled out at a constant velocity of $\sim 3 \mu \mathrm{m} / \mathrm{sec}$. Beads either remained at the cell surface or a tether was formed. Tethers are elastic membrane extensions that have been shown previously to form from cell membranes as well as from pure lipid bilayers, indicating that cytoskeletal components are not necessary for their formation (Hochmuth et al., 1982; Waugh, 1982; Schmidt et al., 1993). All experiments were performed at $\sim 3 \mu \mathrm{m}$ above the slide surface to minimize viscous coupling to the glass surface according to the trap calibration in the perpendicular direction (J. Dai and M. P. Sheetz, unpublished observations). For these experiments, laser trapping was performed on three regions of the extending neurite: the axon, the central region of the growth cone, and the growth cone periphery (Fig. 1).

Trapping results are reported as the percentage of total trapping events, excluding negative encounters, that resulted in either cytoskeletal attachment or tethering. This procedure eliminates operational uncertainty in controlling z-axis position of the latex bead with the optical tweezers since beads that were not lowered sufficiently on the cell surface, and could not make contact (negative encounters), were not included in the reported results. Confidence limits for percentages were calculated at the $95 \%$ level using standard statistical methods (Croxton, 1953; Wagner, 1992) as already described (Schmidt et al., 1993).

Growth cone selection. Growth cones selected for observation were actively growing, isolated from other cells, and possessed a moderate number of filopodia (5-7 on average). Gold particle tracking experiments were performed on the thin, organelle-free lamellar region of the growth cone. Laser optical trapping experiments were conducted on the axon, base, central region, or the periphery of the growth cone (Fig. 1).

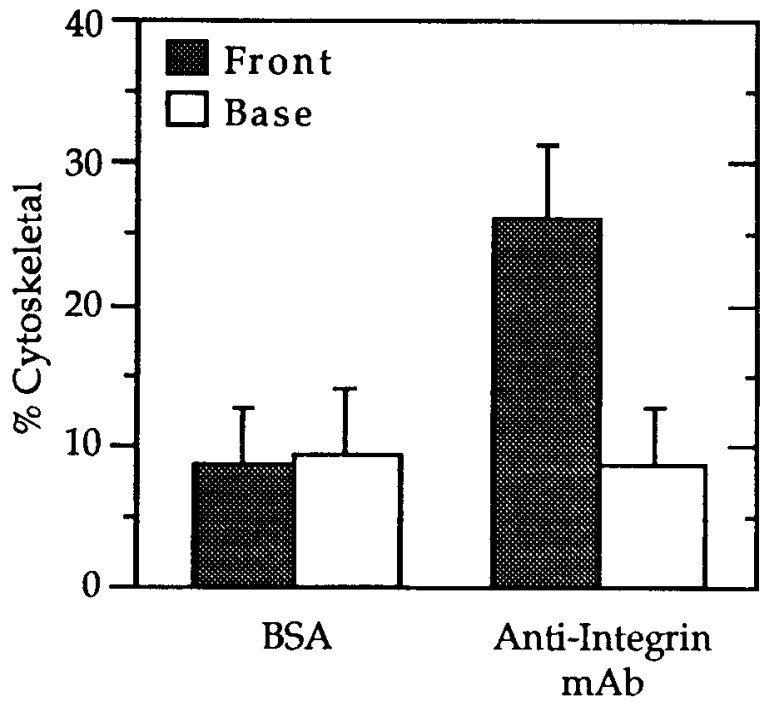

Figure 2. Coupling of integrin occurs preferentially to cytoskeleton at front of motile growth cones. The $0.5 \mu \mathrm{m}$ latex beads, coated either with ES66 anti- $\beta 1$ integrin $\mathrm{mAb}$ or with BSA, were trapped using the laser trap $(\sim 50-60 \mathrm{~mW})$ and held on the growth cone surface for 10 sec (front and base locations for trapping are illustrated in Fig. 1). After release, the bead was scored as either a negative encounter, cytoskeletalassociated, or non-cytoskeletal-attached (as described in Materials and Methods). Motile growth cones display a statistically enhanced $(P<$ $0.01)$ cytoskeletal association of integrin at the cell front compared to either the base of the growth cone or to background levels of interactions (BSA). The percentage of cytoskeletal-attached beads was calculated as the ratio of cytoskeletal-attached beads relative to $n$, the total number of trapping events, excluding negative encounters. Values for $n$ : BSA, 40 (front) and 34 (base); ES66 anti- $\beta 1 \mathrm{mAb}, 92$ (front) and 49 (base).

The growth cone periphery (leading edge) was defined as active lamellar regions and portions of the membrane between extending filopodia (within $\sim 1-2 \mu \mathrm{m}$ of the edge). Trapping could not be performed directly on the filopodia because they are very thin, making it difficult to avoid unwanted attachment of the bead to the glass coverslip. The base of the growth cone was identified as the proximal region of the growth cone separating the actin-rich filopodia and lamellipodia from the microtubule-rich axon shaft.

\section{Results}

\section{Large integrin aggregates couple better to cytoskeleton at leading edge}

Laser tweezers were used to place polystyrene beads coated with an anti-integrin mAb (ES66) on either the front or base (Fig. 1) surface of motile growth cones. Each bead was held on the surface for $10 \mathrm{sec}$ and then scored as either a negative encounter, cytoskeletal-attached, or non-cytoskeletal-attached, based on further manipulation with the trap. Beads that diffused away from the cell surface upon release from the trap were scored as negative encounters. Surface-bound beads that could not be moved within the membrane by the trap were scored as cytoskeletalattached, while those that could be manipulated were scored as non-cytoskeletal-attached. Results are reported as the percentage of cytoskeleton attachment events relative to the total number of bound beads, that is, excluding negative encounters.

Trapping experiments demonstrate a substantial increase $(P<$ $0.01)$ in the probability of cytoskeletal binding to the front periphery ( $26 \pm 5 \%, n=92$ ) versus the base $(8 \pm 4 \%, n=49$ ) of motile growth cones (Fig. 2). The levels of binding at the base of the growth cones were indistinguishable from nonspecific levels of binding with BSA-coated beads (front: $8 \pm 4 \%$, 
$n=40$; base: $9 \pm 5 \%, n=34$ ). Furthermore, there were no spatial differences (front, central region, or axon) in binding of rat IgG-coated beads (data not shown).

Beads that bound to the cell via cytoskeletal linkages (as assayed above) were monitored over longer periods of time and were found to move centripetally loward the base of the growth cone at a rate of $2.1 \pm 1.2 \mu \mathrm{m} / \mathrm{min}( \pm \mathrm{SD}, n=24$ ) relative to the substratum. Once reaching either the center or base of the growth cone, most beads were internalized by the cell.

The slow retrograde motion of latex particles likely represents a stable linkage of large integrin aggregates to rearward flowing cortical actin, as has been described for other cross-linked surface proteins (Dembo and Harris, 1981; Fisher et al., 1988). The rate of rearward flow, $v=2.1 \mu \mathrm{m} / \mathrm{min}$, corresponds well to measurements of retrograde velocity for actin-based microspikes, 1.4-3.6 $\mu \mathrm{m} / \mathrm{min}$ (Bray and Chapman, 1985), supporting a proposed linkage of the integrin to actin. In addition, this rearward velocity is roughly equivalent to the rate of outgrowth for chick sensory neurons ( $\sim 1 \mu \mathrm{m} / \mathrm{min}$; Bray and Chapman, 1985).

\section{Cytoskeleton support of membrane is greater at growth cone} periphery

Laser tweezers were used to place polystyrene beads coated with rat IgG on the surface of the neurite's leading edge, central region or axon (Fig. 1). Each bead was held on the surface for 5 sec and then pulled away from the cell surface at a constant velocity. Beads either remained bound or formed elastic membrane tethers. Results are reported as the percentage of tethers relative to the total number of bound beads, that is, excluding negative encounters.

The results for these trapping experiments demonstrate a spatial dependence for the probability of membrane tether formation (Fig. 3). Many fewer tethers were formed at the leading edge $(34 \pm 3 \%, \pm \mathrm{SD} ; n=98)$ compared to either the central portion of the growth cone $(47 \pm 3 \%, \pm \mathrm{SD} ; n=128)$ or the axon itself (43 $\pm 4 \%, \pm \mathrm{SD} ; n=284$ ). Tethering of the membrane on the leading edge is statistically $(P<0.01)$ lower than tethering found for the central region and the axon. These data suggest that the leading edge of the growth cone is supported to a greater extent by an actin-dependent cytoskeleton that can resist mechanical tether formation.

\section{Small aggregates of integrin undergo directed transport to leading edge}

Gold particles tagged with either anti- $\beta 1$ integrin mAbs (ES66) or BSA were allowed to interact with the dorsal surfaces of motile chick DRG growth cones. Individual beads and small aggregates of beads (aggregate diameter $<260 \mathrm{~nm}$, the limit of resolution of the light microscope) were tracked on the lamellipodia of growth cones for $100 \mathrm{sec}$ using nanometer-precision video microscopy and image analysis. Displacements of beads for the total tracking time were compared to those predicted for pure diffusion using the statistical analysis described in Materials and Methods. A particle track with a low probability $[P(x)$ $<0.05]$ that diffusion could give rise to the displacement of the bead is considered a directed rather than diffusive event.

Dynamic gold bead behavior on the lamellipodial surfaces of motile growth cones is characterized by either diffusion or brief periods of directed transport followed by diffusion (Fig. 4). The paths of the particles show distinct directed excursions or "jumps" (average instantaneous velocity, $v_{i}=43 \pm 16$ $\mu \mathrm{m} / \mathrm{min}, \pm \mathrm{SD} ; n=17$ ) predominantly toward the growth cone

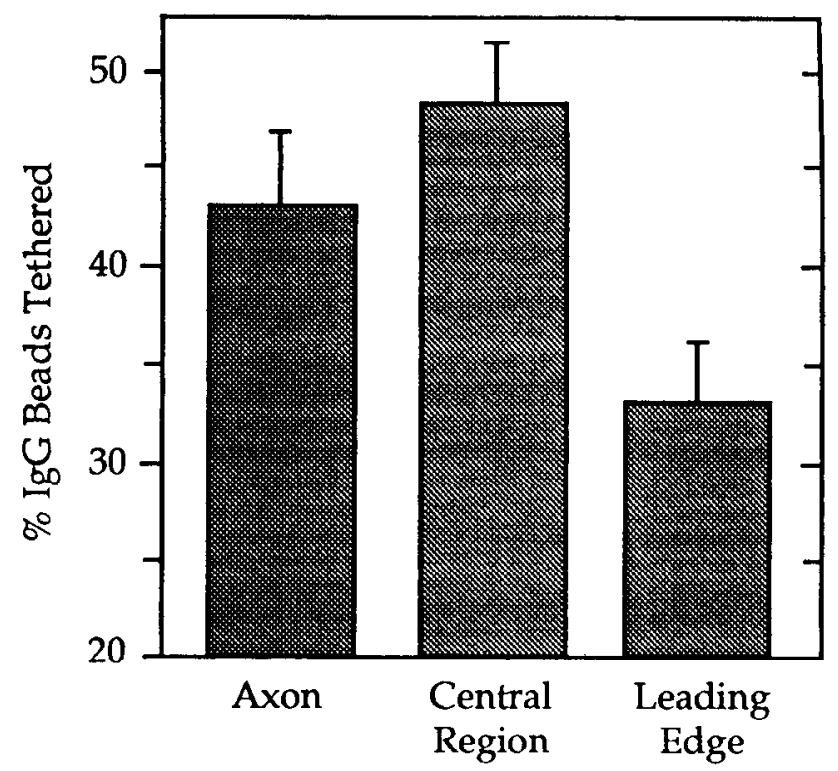

Figure 3. Cytoskeleton support of membrane is greater at the leading cdge. The $0.5 \mu \mathrm{m}$ latex bcads, coated with rat IgG, were trapped using $\sim 60 \mathrm{~mW}$ laser power and held on the surface for $5 \mathrm{sec}$ (locations for trapping are illustrated in Fig. 1). Surface-bound beads were then pulled at a constant velocity, $-3 \mu \mathrm{m} / \mathrm{sec}$, away from the cell surface; beads either remained firmly adhered to the cell surface or formed elastic membrane tethers. Tethering of the membrane on the leading edge is statistically $(P<0.01)$ lower than tethering found for the central region and the axon. The percentages of tethers were calculated relative to the total number of surface attached beads $(n)$. Values for $n$ : axon, 98; central region, 128; leading edge, 284.

periphery interspersed with periods of diffusion $(D=5.1 \pm 3.8$ $\times 10^{-10} \mathrm{~cm}^{2} / \mathrm{sec}, \pm \mathrm{SD} ; n=20$ ). The time-averaged velocity for integrin transport over the entire path, as determined from mean-square-displacement data, is $\langle v\rangle=3.5 \pm 3.5 \mu \mathrm{m} / \mathrm{min}$ $( \pm \mathrm{SD}, n=10)$. This quantity represents the average velocity of integrin movement assuming a constant external force and indicates the degree of directedness for a given path.

For motile growth cones, $50 \pm 11 \%(n=20)$ of the tracked anti-integrin mAb-coated beads underwent directed transport, compared to $15 \pm 10 \%(n=13)$ for BSA-coated particles (Fig. $5)$. The difference between directed transport levels for integrin and background (BSA) is statistically distinct $(P<0.02)$.

These results suggest that integrin in the growth cone is likely transported by an active mechanism to the cell periphery where it is potentially used in the formation of new adhesive contacts. This transport is characterized by diffusion as well as brief periods of highly directed excursions, indicating a transient and reversible interaction of the integrin with a moving cytoskeletal element.

\section{Discussion}

During development of the nervous system, the distal tip of the clongating axon, the growth cone, actively migrates along a precise path over extended distances to innervate the appropriate target tissue. The ability of the growth cone to perceive extracellular guidance cues and to alter its migration path rapidly and accurately is fundamental to the neuronal pathfinding process. Guidance information such as soluble chemoattractants (e.g., NGF) (Gunderson and Barrett, 1980) or chemorepellents (Pini, 1993), as well as insoluble signals located on other cells or in the extracellular matrix (Rutishauser, 1993), is first perceived 


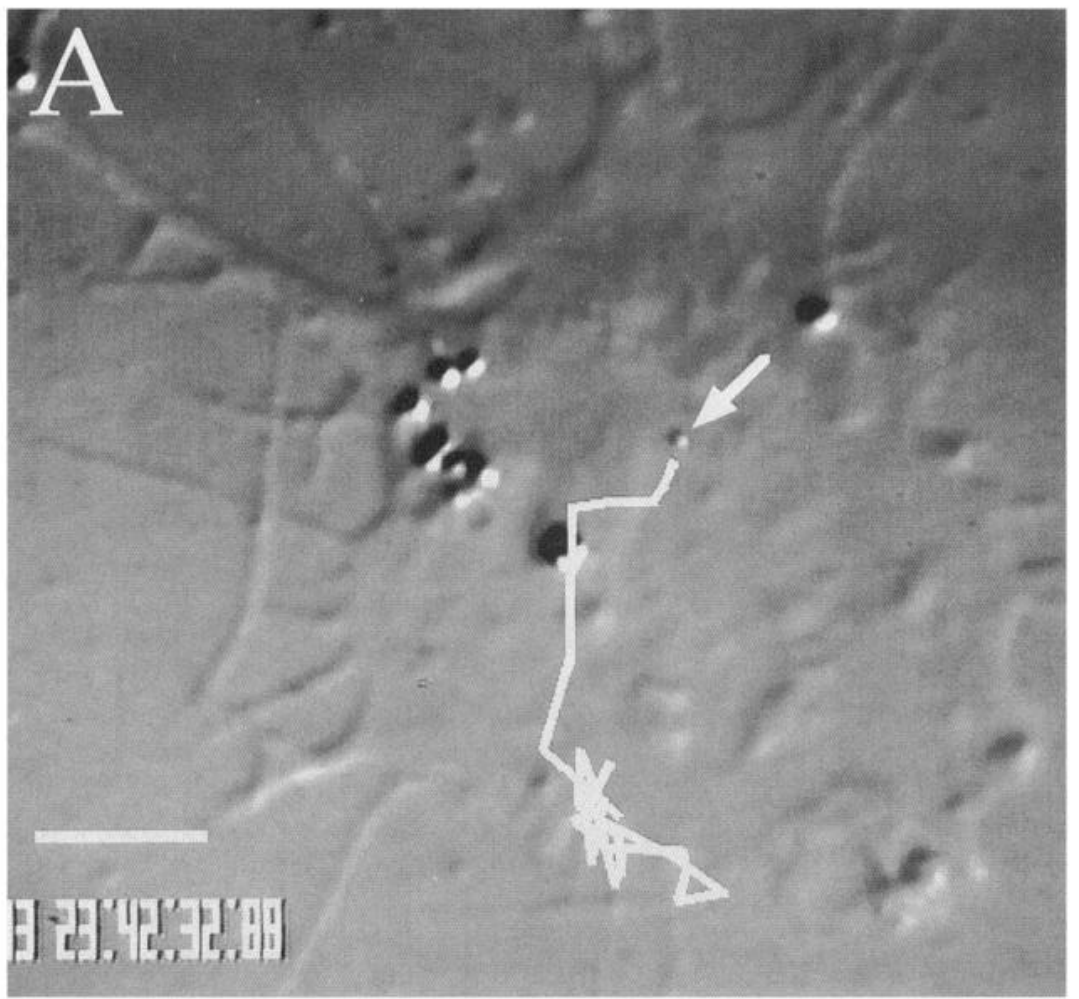

Figure 4. Examples of trajectories for integrins undergoing directed and diffusive motions on the surfaces of neuronal growth cones. The $40 \mathrm{~nm}$ gold beads, coated with ES66 (anti- $\beta 1$ integrin $\mathrm{mAb}$ ), were allowed to interact with the surfaces of migrating chick growth cones. Image analysis techniques were used to track the beads, yielding $\mathrm{x}, \mathrm{y}$-coordinates for the bead position as a function of time. The arrows in $A$ and $B$ indicate the initial positions of gold beads and the line sketches indicate the paths of the particles tracked for a total of $100 \mathrm{sec}$ at $2 \mathrm{sec}$ intervals. In $A$, the bead moves outward toward the growth cone periphery in a series of directed excursions $\left(v_{i}=43 \pm 16 \mu \mathrm{m} / \mathrm{min}, \pm \mathrm{SD} ; n\right.$ $=17)$ and diffusion $(D=5.1 \pm 3.8$ $\times 10^{-10} \mathrm{~cm}^{2} / \mathrm{sec}, \pm \mathrm{SD} ; n=20$ ). In $B$, a bead on a different growth cone undergoes diffusion, resulting in a smaller net displacement than that in $A$. Scale bar, $5 \mu \mathrm{m}$.

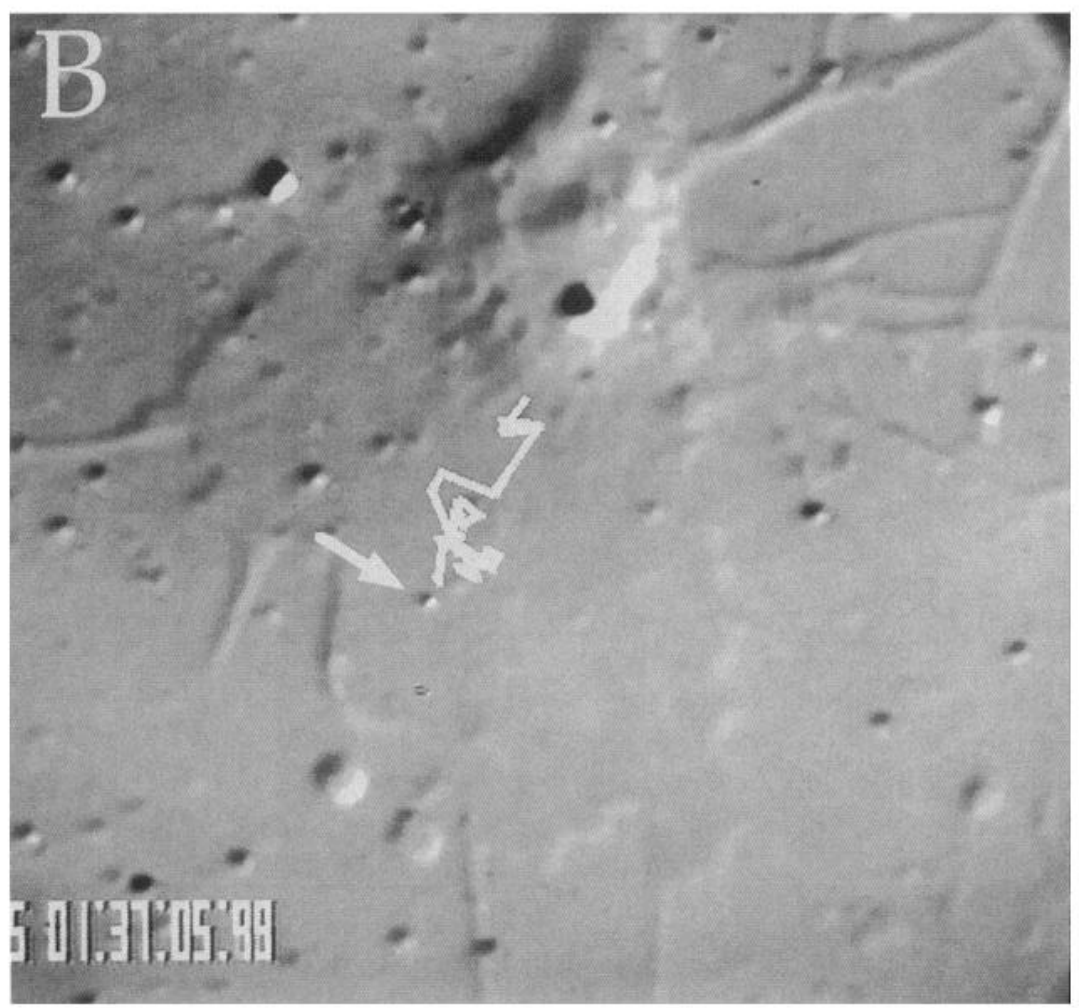

and processed by filopodia and lamellipodia at the growth cone's leading edge. These signals function chemically and/or mechanically to facilitate the reorganization of the actin-based cytoskeleton in the growth cone and the microtubules in the associated axon (Gordon-Weeks, 1987; Sabry et al., 1991; Tanaka and Kirschner, 1991).

Some of the events culminating in the turning of the growth cone, although likely initiated by distinct "steering" signals, may be essentially inseparable from the processes that give rise to net migration of the growth cone. For instance, tension generated within growth cone could serve to drive the forward motion of the growth cone and subsequent axonal elongation (Bray, 1984; Zheng et al., 1991) as well as to modify the direction in which new filopodial processes are formed (Bray, 1979). Understanding how this tension is regulated could provide insights into two of the fundamental properties of neuronal pathfinding: 


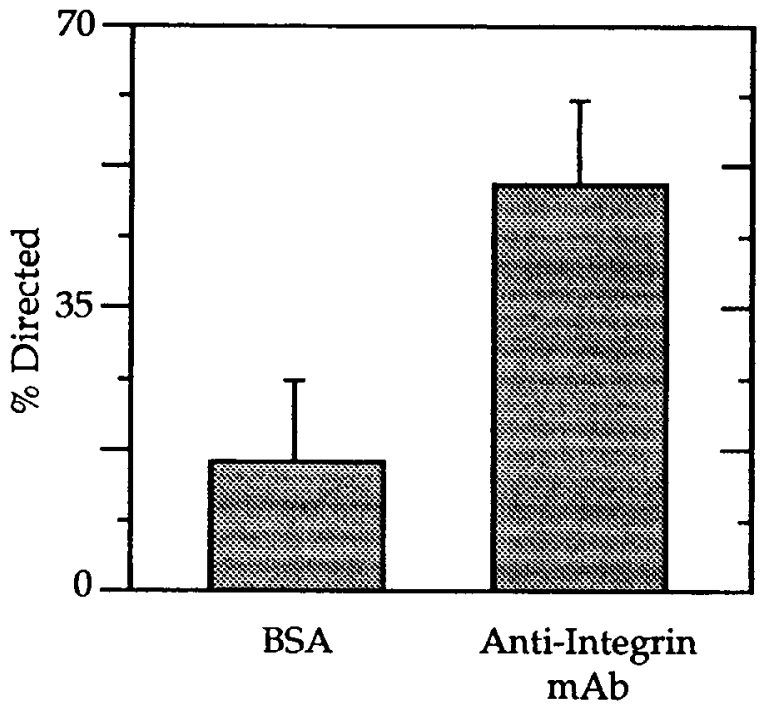

Figure 5. Fraction of $\beta 1$ integrin that undergoes rapid, directed transport on the surfaces of motile growth cones. The $40 \mathrm{~nm}$ gold beads, coated either with anti- $\beta 1$ integrin mAb (ES66) or with BSA, were allowed to interact with the surfaces of motile chick DRG growth cones. The directed versus diffusive behavior for each bead was determined statistically as described in Materials and Methods. The percentage of beads that underwent directed transport is shown for BSA- and ES66coated beads. Beads coated with anti- $\beta 1$ integrin $\mathrm{mAb}$ (ES66) displayed statistically higher levels $(P<0.02)$ of directed transport compared to beads coated with BSA. Total number of beads tracked: ES66, 20; BSA, 13.

growth cone migration and guidance. Our finding that integrin interacts preferentially with the cytoskeleton at the front versus the base of the growth cone suggests that this regulation may occur at the level of integrin-cytoskeleton interactions. Stronger associations of the integrin adhesion receptor with cytoskeleton components at the leading edge likely modulate tension in the growth cone and hence alter net migration and neurite turning behavior.

With respect to forward motion, more prominent interactions of integrin with actin, the locomotory apparatus, at the growth cone periphery provide for a net transmission of force at the cell front, thereby permitting forward locomotion. In other words, force generated by the actin cytoskeleton (likely via an actinmyosin interaction) is transmitted as traction along the underlying substrate only through linked integrins. Thus, enhanced linkage of integrins at the cell front permits a larger transduction of force that can subsequently overcome weak attachments at the base of the growth cone to pull the growth cone and axon forward.

Altered integrin-cytoskeleton interactions may also serve to modulate growth cone steering. Bray (1979) experimentally demonstrated that chick dorsal root ganglion growth cones advance in the direction of greatest tension, with tension being the force generated between the growth cone and its associated axon. Amputated growth cones, relieved of mechanical stress, tended to branch extensively in all directions after surgery. In addition, manual micromanipulation of the neurite to displace it to one side resulted in a directional change such that the growth cone proceeded to move in a straight line with respect to the axon (i.e., in the direction of greatest tension). This stress is generated by firm cell-cell or cell-matrix adhesive interactions of growth cone lamellipodia and/or filopodia, as mediated by
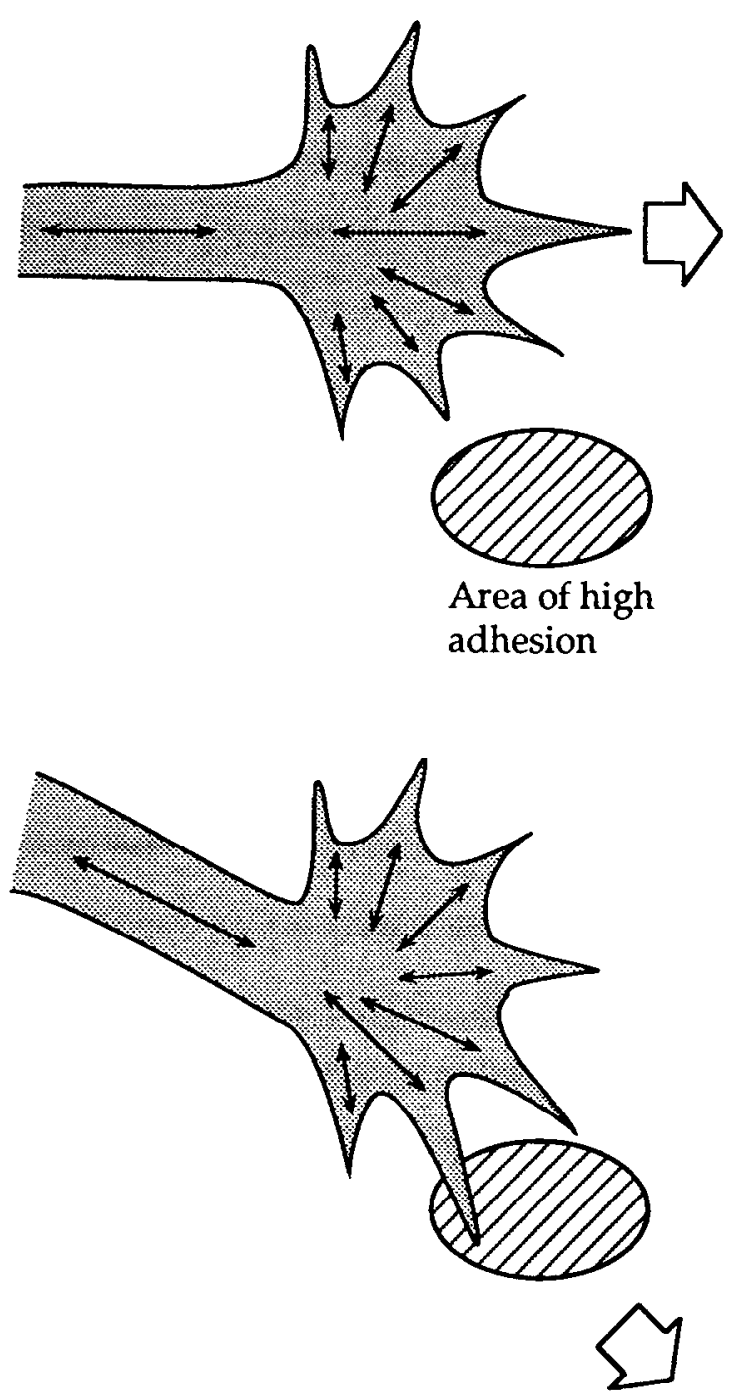

Figure 6. Model for how the lack of adhesive interactions at growth cone base can aid in turning events. Shown are schematic illustrations of a growth cone elongating to the right, and its subsequent migration downward after encountering a highly adhesive substratum. The absence of adhesions at the back of the growth cone, as mediated by a lack of integrin-cytoskeleton coupling (Fig. 2) and/or reduced cytoskeleton support of the membrane (Fig. 3), permits changes in adhesion at the leading edge to be rapidly sensed by the axon as tension. As a result, turning of the elongating axon follows reorientation of filopodial and lamellipodial attachment. The double-headed arrows indicate the direction and magnitude of tension in the neurite, and the larger open arrows indicate the path of axonal elongation.

adhesion receptors such as the integrins. Contacts at the leading edge, and not the base of the growth cone, provide the best explanation for Bray's finding that growth cones move in a straight path with respect to the axon (Fig. 6). In this manner, strong adhesions of growth cone filopodia to favorable substratum are "felt" by the axon, and reorganization along this new path proceeds more quickly than if adhesions existed at the base of the growth cone. Therefore, regulation of adhesive events at the integrin-cytoskeleton level is critical not only for migration but also for generating the tension necessary to permit either persistent growth cone advancement or cell turning.

Consistent with the potential role of integrin-cytoskeleton associations in asymmetrical force transmission and tension generation is our finding that the membrane at the leading edge has 
a lower tendency to form tethers. An enhanced ability to form elastic membrane tethers is likely indicative of an "unsupported" membrane (Schmidt et al., 1993), with support referring to the close association of membrane with a cytoskeletal network resistant to mechanical deformation. The lack of membrane support in proximal regions of the growth cone and in the axon could provide another means by which an asymmetry in traction is gencrated within the active neurite. For instance, the physical separation of the membrane from the cytoskeletal network in these regions may reduce the potential for the formation of effective adhesions via transmembrane adhesion receptors, generating a regional difference in force transduction. This rationale may in part explain the inability of integrin to associate with the actin cytoskeleton at the base of the growth cone. In any event, as described above, lack of interactions at the base of the growth cone could contribute to the axonal tension necessary for turning events.

In addition to an asymmetry in traction forces, growth cone guidance also requires that adhesion receptors be supplied to the periphery of the cell's leading edge where nascent contacts are formed. We used nanometer-precision motion analysis to show that integrin undergoes a rapid, directed transport preferentially to the leading edge of the growth cone where presumably new contacts are initiated. Directed transport consists of periods of "jumps", with an instantaneous velocity of about $40 \mu \mathrm{m} / \mathrm{min}$, followed by either pure diffusion or diffusion with an imposed velocity, suggesting that integrin interacts transiently with moving cytoskeletal elements (Kucik et al., 1989; Schmidt et al., 1993). The observed rapid, directed motions on growth cones are statistically discernible from diffusion and are unlikely the result of unusual diffusion patterns for reasons previously outlined (Schmidt et al., 1994). The mechanism for the rapid, directed motions is not known, but one hypothesis is that integrin couples indirectly to a subset of short actin filaments that are transported forward along the underlying actin filament network by a motor protein (e.g., a myosin I analog) (Sheetz et al., 1990). Regardless of the mechanism, such motions could likely play a physiological role in growth cone migration by providing integrins to the cell's advancing edge where adhesions are initiated. In addition, the overall speed of integrin delivery to the leading edge, as mediated by directed transport, may be critical for the turning of the growth cone and its axon. Since filopodia are rapidly and constantly "sampling" the environment over which the neurite is moving, it is necessary that integrins be supplied to all future areas of attachment within a relatively short time frame to permit accurate pathfinding.

The similarities between growth cone migration and fibroblast migration are striking. Although previous comparisons of morphological behavior and cytoskeletal dynamics have becn made (Trinkaus, 1985), the observations reported here point to common mechanisms for regulation of locomotion and cell turning at the level of integrin-cytoskeleton associations (Schmidt et al., 1993).

\section{References}

Bentley D, O'Connor TP (1994) Cytoskeletal events in growth cone steering. Curr Opin Neurobiol 4:43-48.

Berg HC (1983) Random walks in biology, pp 14-16. Princeton, NJ: Princeton UP.

Bozyczko D, Horwitz AF (1986) The participation of a putative cell surface receptor for laminin and fibronectin in peripheral neurite extension. J Neurosci 6:1241-1251.
Bray D (1979) Mechanical tension produced by nerve cells in tissue culture. J Cell Sci 37:391-410.

Bray D (1984) Axonal growth in response to experimentally applied tension. Dev Biol 102:379-389.

Bray D (1987) Growth cones: do they pull or are they pushed? Trends Neurosci 10:431-434.

Bray D, Chapman K (1985) Analysis of microspike movements on the neuronal growth cone. J Neurosci 5:3204-3213.

Bray D, Hollenbeck PJ (1988) Growth cone motility and guidance. Annu Rev Cell Biol 4:43-61.

Croxton FE (1953) Elementary statistics with applications in medicine and the biological sciences. New York: Dover.

Cunningham BA, Hemperly JJ, Murray BA, Prediger EA, Brackenbury R, Edelman GM (1987) Neural cell adhesion molecule: structure, immunoglobulin-like domains, cell surface modulation and alternative splicing. Science 236:799-806.

Dembo M, Harris A (1981) Motion of particles adhering to the leading lamella of crawling cells. J Cell Biol 91:528-536.

Duband J-L, Nuckolls GH, Ishihara A, Hasegawa T, Yamada KM, Thiery JP, Jacobson K (1988) Fibronectin receptor exhibits high lateral mobility in embryonic locomoting cells but is immobile in focal contacts and fibrillar streaks in stationary cells. J Cell Biol 107:13851396.

Fisher GW, Conrad PL, DeBiasio RL, Taylor DL (1988) Centripetal transport of cytoplasm, actin and the cell surface in lamellipodia of fibroblasts. Cell Motil Cytoskel 11:235-247.

Forscher P, Smith SJ (1988) Actions of cytochalasins on the organization of actin filaments and microtubules in a neuronal growth cone. J Cell Biol 107:1505-1516.

Gelles J, Schnapp BJ, Sheetz MP (1988) Tracking kinesin-driven movements with nanometre-scale precision. Nature 331:450-453.

Gordon-Weeks PR (1987) The cytoskeletons of isolated neuronal growth cones. Neurosci 21:977-987.

Gordon-Weeks PR (1989) Growth at the growth cone. Trends Neurosci 12:238-240.

Gordon-Weeks PR (1991) Evidence for microtubule capture by filopodial actin filaments in growth cones. Neuroreport 2:573-576.

Gunderson RW, Barrett JN (1980) Characterization of the turning response of dorsal root neurites toward nerve growth factor. J Cell Biol 87:546-554.

Heidemann SR, Lamoureux P, Buxbaum RE (1990) Growth cone behavior and production of traction force. J Cell Biol 111:1949-1957.

Hochmuth RM, Wiles HC, Evans EA, McCown JT (1982) Extensional flow of erythrocyte membrane from cell body to elastic tether: II. Experiment. Biophys J 39:83-89.

Koch GLE (1980) Microfilament-membrane interactions in the mechanism of capping. Symp Br Soc Cell Biol 3:410-444.

Kucik DF, Elson EL, Sheetz MP (1989) Forward transport of glycoproteins on leading lamellipodia in locomoting cells. Nature 340: 315-317.

Kucik DF, Kuo SC, Elson EL, Sheetz MP (1991) Preferential attachment of membrane glycoproteins to the cytoskeleton at the leading edge of lamella. J Cell Biol 114:1029-1036.

Kuo SC, Gelles J, Steuer E, Sheetz MP (1991) A model for kinesin movement from nanometer-level measurements of kinesin and cytoplasmic dynein and force measurements. J Cell Sci 14:135-138.

Letourneau PC (1981) Immunocytochemical evidence for colocalization in neurite growth cones of actin and myosin and their relationship to cell-substratum adhesions. Dev Biol 85:113-122.

Lin C-H, Forscher P (1993) Cytoskeletal remodeling during growth cone-target interactions. J Cell Biol 121:1369-1383.

Mitchison T, Kirschmer M (1988) Cytoskeletal dynamics and nerve growth. Neuron 1:761-772.

O'Connor TP, Bentley D (1993) Accumulation of actin in subsets of pioneer growth cone filopodia in response to neural and epithelial guidance cues in situ. J Cell Biol 123:935-948.

Okabe S, Hirokawa N (1991) Actin dynamics in growth cones. J Neurosci 11:1918-1929.

Okun LM (1972) Isolated dorsal root ganglion neurons in culture: cytological maturation and extension of electrically active processes. J Neurobiol 3:111-151.

Pini A (1993) Chemorepulsion of axons in the developing mammalian central nervous system. Science 261:95-98.

Reichardt LF, Tomaselli KJ (1991) Extracellular matrix molecules and 
their receptors: functions in neural development. Annu Rev Neurosci $14: 531-570$.

Rutishauser U (1993) Adhesion molecules of the nervous system. Curr Opin Neurobiol 3:709-715.

Sabry JH, O'Connor TP, Evans L, Toroian-Raymond A, Kirschner M, Bentley D (1991) Microtubule behavior during guidance of pioneer growth cones in situ. J Cell Biol 115:381-395.

Schmidt CE, Horwitz AF, Lauffenburger DA, Sheetz MP (1993) Integrin-cytoskeletal interactions in migrating fibroblasts are dynamic, asymmetric, and regulated. J Cell Biol 123:977-991.

Schmidt CE, Chen T, Lauffenburger DA (1994) Simulation of integrincytoskeletal interactions in migrating fibroblasts. Biophys J 67:461474.

Seilheimer B, Schachner M (1988) Studies of adhesion molecules mediating interactions between cells of peripheral nervous system indicate a major role for $\mathrm{L} 1$ in mediating sensory neurons growth on Schwann cells in culture. J Cell Biol 107:341-351.

Sheetz MP, Turney S, Qian H, Elson EL (1989) Nanometre-level analysis demonstrates that lipid flow does not drive membrane glycoprotein movements. Nature 340:284-288.

Sheetz MP, Baumrind NL, Wayne DB, Pearlman AL (1990) Concen- tration of membrane antigens by forward transport and trapping in neuronal growth cones. Cell 61:231-241.

Takeichi M (1988) The cadherins: cell-cell adhesion molecules controlling animal morphogenesis. Development 102:639-655.

Tanaka EM, Kirschner MW (1991) Microtubule behavior in the growth cones of living neurons during axon elongation. J Cell Biol 115:345 363.

Tessier-Levigne M, Placzek M (1991) Target attraction: are developing axons guided by chemotropism? Trends Neurosci 15:303-310.

Trinkaus JP (1984) Cells into organs. The forces that shape the embryo. Fnglewood Cliffs, NII: Prentice-Hall.

Trinkaus JP (1985) Further thoughts on directional cell movement during morphogenesis. J Neurosci Res 13:1-19.

Wagner SF (1992) Introduction to statistics. New York: Harper Collins.

Walsh FS, Doherty P (1991) NCAM gene structure and function. Semin Neurosci 3:271-284.

Waugh RE (1982) Surface viscosity measurements from large bilayer vesicle tether formation: II. Experiments. Biophys J 38:29-37.

Zheng J, Lamoureux P, Santiago V, Dennerll T, Buxbaum RE, Heidemann SR (1991) 'lensile regulation of axonal elongation and initiation. J Neurosci 11:1117-1125. 